\title{
Differences in Blood Pressure Levels Among Children by Sociodemographic Status
}

\author{
Melissa Goulding, $\mathrm{MS}^{1}$; Robert Goldberg, $\mathrm{PhD}^{2}$; Stephenie C. Lemon, $\mathrm{PhD}^{1}$
}

Accessible Version: www.cdc.gov/pcd/issues/2021/21_0058.htm

Suggested citation for this article: Goulding M, Goldberg R, Lemon SC. Differences in Blood Pressure Levels Among Children by Sociodemographic Status. Prev Chronic Dis 2021;18:210058. DOI: https://doi.org/10.5888/pcd18.210058.

\section{PEER REVIEWED}

\section{Summary}

What is already known on this topic?

High blood pressure (BP) affects many US children; however, most prevalence estimates are based on outdated data and guidelines. Although studies have shown that childhood hypertensive BP is not evenly distributed across sociodemographic groups, they do not account for body weight as a contributor to prevalence disparities.

\section{What is added by this report?}

Our study provides contemporary national prevalence estimates of elevated and hypertensive BP among children across sociodemographic groups and examines the effect of weight on observed disparities.

What are the implications for public health practice?

Factors beyond inequalities in body weight may contribute to disparities in elevated BP among US children. Further investigation of these disparities is needed to inform targeted public health efforts.

\section{Abstract}

\section{Introduction}

The American Academy of Pediatrics (AAP) updated its blood pressure (BP) screening guidelines in 2017 to emphasize body weight as a risk factor. We provide contemporary, nationally representative estimates of prevalence of elevated and hypertensive BP among US children and examine sociodemographic prevalence differences, accounting for the influence of weight.

\section{Methods}

We used cross-sectional data from children aged 8 to 17 years $(\mathrm{N}$ $=5,971$; weighted $\mathrm{N}=36,612,323$ ) collected from 2011 through 2018 in 4 biennial cycles of the National Health and Nutrition Examination Survey (NHANES). Children's BP was categorized as normal, elevated, or hypertensive. Sociodemographic characteristics included were sex, age, race/ethnicity, family income, and education. Log binomial regression, with and without adjustment for weight (dichotomized at the 85th body mass index percentile), determined prevalence estimates and differences for elevated and hypertensive BPs with $95 \%$ CIs.

\section{Results}

In NHANES data collected from 2011 through 2018, 7.2\% (95\% CI, $6.3 \%-8.3 \%$ ) of US children had elevated BP, and 3.8\% (95\% CI, 3.3\%-4.5\%) had hypertensive BP according to 2017 AAP guidelines. Differences in prevalence of weight-adjusted elevated BP indicated higher prevalence among children aged 16 to 17 years compared with children aged 8 to 9 years (prevalence difference, $+6.3 \%$; 95\% CI, 3.2\%-9.4\%), among males compared with females $(+4.6 \%$; $95 \% \mathrm{CI}, 2.7 \%-6.4 \%)$, and among non-Latino Black children compared with non-Latino White children $(+4.0 \%$; 95\% CI, 2.2\%-5.8\%). Crude hypertensive BP prevalence was highest among children aged 8 to 9 years, male children, and Mexican American children. The only difference remaining after weight adjustment was among children aged 8 to 9 years and 13 to 15 years.

\section{Conclusion}

Elevated BP was most prevalent among US children who were older, male, or non-Latino Black. Factors beyond inequalities in body weight may contribute to disparities in elevated BP.

\section{Introduction}

Hypertension affected nearly 4\% of US children from 2013 through 2016 (1). The high prevalence of childhood obesity has contributed to an increase in several chronic conditions among children, including hypertension (2). Children who are overweight have higher systolic and diastolic blood pressure (BP) (3) than normal-weight children, and those with obesity have a threefold higher risk of hypertension compared with children of healthy weight (4). Given the relationship between weight and BP, the American Academy of Pediatrics (AAP) changed its clinical practice guidelines in 2017 with new normative pediatric BP 
tables to assess children's BP percentiles and categories on the basis of healthy body weight, in contrast to their previous guidelines, which included children of all weight statuses (5). Prevalence estimates based on AAP's earlier guidelines may have been biased by body weight and therefore warrant reinvestigation. Although AAP's guideline changes increased estimated prevalence of hypertension among US children (from $1.9 \%$ to $3.5 \%$ ) (1), national estimates beyond 2016 are unavailable $(1,5,6)$.

Few studies have described sociodemographic factors associated with hypertension among US children. Although prevalence in those studies appears to be higher among males and among Black, Mexican American, and other Latino children (1,5,7-9), many of those studies were based on past AAP guidelines (10) and few investigated the extent to which disparities in BP could be explained by differences in weight $(7,9)$. Furthermore, investigation of potential associations between hypertension and socioeconomic factors has been limited $(11,12)$.

The objective of our study was to provide nationally representative prevalence estimates of elevated and hypertensive BP among US children according to 2017 AAP guidelines. We also examined sociodemographic differences in prevalence and explored the role of weight status in relationship to differences in BP levels.

\section{Methods}

\section{Study design and database}

Our cross-sectional study used nationally representative data from the National Health and Nutrition Examination Survey (NHANES) (13), which is collected biennially by the National Center for Health Statistics to provide data on the health status of community-dwelling US residents. NHANES collects sociodemographic, dietary, and general health information by survey and medical, dental, and laboratory data by physical examination. We used data from 2011-2018, which consists of 4 biennial cycles. Unweighted survey response rates ranged from $53.6 \%$ to $78.5 \%$ for our study sample. Additional adjustments to weighting procedures were used to reduce the potential effects of response bias resulting from a lower response rate in the 2017-2018 NHANES cycle (13). NHANES data collection is approved by the National Center for Health Statistics Research and Ethics Review Board. Participant and parental consent were obtained for children aged 13 years or older. Participant assent and parental consent were obtained for children aged 7 to 12 years.

\section{Study population}

NHANES BP data comes from physical examinations (13). For our study we included children aged 8 to 17 years for whom data on BP, height, weight, race/ethnicity, and socioeconomic characteristics were available. We excluded children who were missing BP measurements $(\mathrm{n}=338)$, had fewer than 3 BP readings $(\mathrm{n}=$ 68 ), were missing data on body mass index (BMI) (weight in $\mathrm{kg}$ / height in $\left.\mathrm{m}^{2}\right)(\mathrm{n}=32)$, or were missing data on sociodemographic characteristics $(\mathrm{n}=702)$. The final sample included 5,971 children, weighted to represent $36,612,323$ children. To provide biennial prevalence estimates of hypertensive and elevated BP, the sample was defined by NHANES cycle. We used the entire sample for prevalence estimates of various BP parameters and differences in these end points according to sociodemographic factors.

Operational definition of pediatric elevated and hypertensive BP. Although clinical diagnosis of hypertension requires BP measurement across at least 3 occasions, NHANES is limited to physical examination on 1 occasion. Therefore, 3 BP measurements taken on a single occasion were averaged for each child in accordance with AAP guidelines for clinicians and common practice in pediatric hypertension studies (1,5,7-9). NHANES BP measurement techniques have been described previously (13). For children aged 8 to 12 years, we used age, sex, and height to determine their BP percentile according to the 2017 AAP BP tables. BP percentiles (for children aged $<13 \mathrm{y}$ ) or average measurement (for children aged 13-17 y) were then used for categorization according to 2017 AAP guidelines. Elevated BP was defined as ranging from $\geq 90$ th percentile to $<95$ th percentile or $120 /<80 \mathrm{~mm} \mathrm{Hg}$ to $<95$ th percentile (whichever is lower) for children aged 8 to 12 years and $120 /<80$ to $129 /<80 \mathrm{~mm} \mathrm{Hg}$ for those aged 13 to 17 years. Hypertensive BP was defined as a BP percentile of $\geq 95$ or an average BP of $\geq 130 / 80 \mathrm{~mm} \mathrm{Hg}$ (whichever was lower) for children aged 8 to 12 years and $\geq 130 / 80 \mathrm{~mm} \mathrm{Hg}$ for those aged 13 to 17 years.

Body mass index percentile. Children's standing height and weight were measured by trained professionals during the NHANES physical examination, and their BMI was calculated. Methods and equipment used for anthropometric measures have been described previously (14). We determined BMI percentiles according to the Centers for Disease Control and Prevention 2000 growth charts (15). Weight status was categorized by BMI percentile to represent healthy weight (BMI percentile $<85$ ), overweight (BMI percentile $\geq 85$ to $<95$ ), and obesity (BMI percentile $\geq 95$ ). For adjusted prevalence estimates, we dichotomized weight to indicate unhealthy weight status (BMI percentile $\geq 85$ ).

Sociodemographic factors associated with elevated and hypertensive BP. Age at the time of the NHANES physical exam-

\footnotetext{
The opinions expressed by authors contributing to this journal do not necessarily reflect the opinions of the U.S. Department of Health and Human Services, the Public Health Service, the Centers for Disease Control and Prevention, or the authors' affiliated institutions.
} 
ination was determined by the child's date of birth and was stratified at 8 to 9 years, 10 to 12 years, 13 to 15 years, and 16 to 17 years. Sex was determined by self-report with options of male or female. We used the more inclusive NHANES race/ethnicity variable in which children who identified as Mexican American were coded as such, those who identified as Hispanic or Latino were coded as other Latino, and those who identified as non-Latino were coded according to self-reported race of White, Black, Asian, or other (American Indian or Alaska Native, Native Hawaiian or Pacific Islander, mixed race).

We used 2 proxy measures for socioeconomic status, family poverty income ratio (PIR) and parent/guardian education level. PIR was calculated by dividing family income by the Department of Health and Human Services' poverty guidelines and then categorized as low (PIR $<1.3$ ), medium (PIR $\geq 1.3$ and $<3.5$ ), and high (PIR $\geq 3.5$ ). This categorization was used to be consistent with past obesity-related research and because a PIR of $<1.3$ is often used to determine eligibility for federally funded programs, including the Supplemental Nutrition Assistance Program (16). Parent/guardian education level was measured as the highest education of the household reference person, who was the first person listed in the household aged 18 years or older who owned or rented the residence.

\section{Statistical analysis}

We computed frequencies on our study sample. Because each of the continuous variables had nonnormal distributions (assessed via Shapiro-Wilk test), medians with interquartile range were calculated. Prevalence estimates of elevated and hypertensive BP were computed for the 2011-2018 period overall and by 4 biennial cycles. We estimated crude prevalence differences and weight status (BMI percentile $\geq 85$ ) adjusted prevalence differences with 95\% CIs for elevated and hypertensive BP for each sociodemographic subgroup through log binomial regression with the identity link (17). Each sociodemographic factor was assessed separately. Models were then adjusted for weight status. Assessment of correlations between weight status and each sociodemographic variable suggested adjusted models were not collinear. All analyses were appropriately weighted and analyzed with examination sample weights and Taylor series linearization (13) accounting for the complex sampling design of NHANES.

\section{Results}

Characteristics of US children. Among children aged 8 to 17 years in NHANES 2011-2018, nearly a third (31.1\%) were aged 13 to 15 years (Table 1$)$. About half were female (49.7\%). More than half $(55.4 \%)$ were non-Latino White. The next largest racial/eth- nic group was Mexican American (14.1\%). Over one-third $(37.6 \%)$ had an unhealthy body weight index (BMI) ( $\geq 85$ percentile).

Prevalence of elevated and hypertensive BP. In the most recent NHANES cycle, 2017-2018, the prevalence of elevated BP was $6.2 \%(95 \%$ CI, 4.2\%-9.3\%) (Table 2 ) and the prevalence of hypertensive BP was 3.9\% (95\% CI, 2.9\%-5.3\%). Prevalence of hypertensive BP overall from 2011-2018 was 3.8\% (95\% CI, $3.3 \%-4.5 \%)$.

Elevated and hypertensive BP by child's weight status. Both elevated and hypertensive BP were more prevalent in children categorized as overweight or as having obesity compared with children of healthy weight. For elevated BP among overweight children, the prevalence difference was $+4.3 \%(95 \% \mathrm{CI}, 1.8 \%-6.8 \%)$. For children with obesity, the prevalence difference for elevated BP was $+7.8 \%(95 \%$ CI, 5.7\%-9.9\%). For hypertensive BP, the prevalence difference for overweight children was $+1.9 \%$ (95\% CI, $0.3 \%-3.5 \%$ ), and for children with obesity, the prevalence difference was $+6.4 \%(95 \%$ CI, 4.3\%-8.6\%) (Table 3). Children with BMIs within the range indicating obesity had a prevalence of hypertensive BP almost 4 times greater than those with healthy weight $(8.6 \% ; 95 \% \mathrm{CI}, 6.9 \%-10.9 \%)$ versus $2.2 \%(95 \% \mathrm{CI}$, $1.7 \%-2.8 \%)$.

Sociodemographic differences in elevated BP prevalence. Prevalence of elevated BP differed across sociodemographic groups. Prevalence was higher among males $(9.6 \% ; 95 \% \mathrm{CI}$, $8.1 \%-11.2 \%)$ than among females $(4.9 \%$; 95\% CI, 3.9\%-6.1\%), and the difference remained significant after adjustment for body weight status (adjusted prevalence difference, $+4.6 \%$; 95\% CI, $2.8 \%-6.5 \%$ ) (Table 3). Prevalence was also greater among older children (16-17 y vs $8-9$ y) before adjustment (crude prevalence difference $+6.9 \% ; 95 \%$ CI, 3.7\%-10.2\%) and after adjustment (adjusted prevalence difference, $+6.3 \%$; 95\% CI, 3.2\%-9.4\%). Children of non-Latino Asian descent had the lowest crude prevalence of elevated BP $(4.6 \% ; 95 \%$ CI, 2.9\%-7.4\%), followed by non-Latino White children $(6.3 \%$; 95\% CI, 5.1\%-7.9\%), whereas non-Latino Black children had significantly greater prevalence $(10.4 \%$; 95\% CI, $8.8 \%-12.1 \%)$, with the crude prevalence difference $+4.0 \%(95 \%$ CI, 2.1\%-5.9\%) (Table 3$)$. After adjustment for weight status, these prevalence differences remained: $+4.0(95 \%$ CI, 2.2\%-5.8\%) among non-Latino Black children compared with non-Latino White children. Elevated BP also appeared to have an inverse relationship with socioeconomic status: the highest prevalence estimates were observed among children of low-income families $(8.4 \%$; 95\% CI, $7.3 \%-9.6 \%)$ or from a household with parent/guardian educational attainment of less than a high school diploma $(8.0 \% ; 95 \% \mathrm{CI}, 6.5 \%-9.9 \%)$ in unadjusted estimates. These socioeconomic differences were attenuated, and significance re-

The opinions expressed by authors contributing to this journal do not necessarily reflect the opinions of the U.S. Department of Health and Human Services, the Public Health Service, the Centers for Disease Control and Prevention, or the authors' affiliated institutions. 
mained only when comparing those with the lowest parent/guardian education (<high school diploma) to those with the highest (college graduate or above) after adjustment for weight status (adjusted prevalence difference, $+2.1 \%$; 95\% CI, $0 \%-4.3 \%$ ).

Sociodemographic differences in prevalence of hypertensive BP. Prevalence of hypertensive BP also differed by sociodemographic groups as did crude and adjusted prevalence differences. Although the unadjusted prevalence estimates were higher among children in all racial/ethnic groups compared with non-Latino White children (unadjusted prevalence difference from $+0.7 \%$ [95\% CI, $-1.2 \%$ to $2.6 \%$ ] to $+2.3[95 \% \mathrm{CI}-0.4 \%$ to $5.1 \%])$, these differences were not significant (Table 3 ). The unadjusted prevalence of hypertensive BP was higher among male children (prevalence, $+1.7 \%$; $95 \% \mathrm{CI}, 0.2 \%-3.2 \%$ ) than female children, but this difference was no longer significant after adjustment for the differential distribution of weight status. The prevalence of hypertensive BP was lower among children aged 13 to 15 years compared with those aged 8 to 9 years (unadjusted prevalence difference, $-4.1 \% ; 95 \% \mathrm{CI},-5.9 \%$ to $-2.3 \%$ ), and these differences remained significant after adjustment for weight status (adjusted prevalence difference, $-3.8 \% ; 95 \% \mathrm{CI},-5.6 \%$ to $-2.0 \%)$. No differences in hypertensive BP prevalence were seen across PIR levels or parent/guardian education levels.

\section{Discussion}

Our study showed prevalence among children aged 8 to 17 years to be $7.2 \%$ for elevated BP and 3.8\% for hypertensive BP according to 2017 AAP guidelines. Our findings also confirm the important relationship between body weight and BP among children aged 17 years or younger. Children who were classified as overweight or having obesity were more likely to have elevated or hypertensive BP than healthy-weight children. We identified associated sociodemographic differences and found that some, but not all, of these differences were attenuated after accounting for disparities in body weight $(1,8,9)$. We found higher prevalence estimates of elevated BP in males, older children (16-17 y), non-Latino Black children, and children of lower socioeconomic status. After adjustment for weight status, elevated BP prevalence differences in age, sex, race/ethnicity, and parent/guardian education persisted in these groups. Hypertensive BP was highest among younger children (8-9 y), Mexican America children, and males.

The prevalence of elevated and hypertensive BP observed in our study is higher than previous estimates $(7,8)$. These earlier estimates were based on previous guidelines where weight distribution skewed the normative tables resulting in higher BPs at lower percentiles and fewer children meeting the elevated and hypertensive percentiles (18). A previous study that used the 2017 AAP guidelines found a declining trend in hypertensive BP prevalence among children aged 8 to 17 years in NHANES data when comparing data collected in 2005-2008 with data collected in 2013-2016 (1). Focusing on more recent data and not aggregating biennial cycles, we found the prevalence of elevated and hypertensive BP to fluctuate between the study years of 2011 and 2018 . However, overlapping confidence intervals suggest these differences were probably due to chance. The prevalence of elevated and hypertensive BPs was highest in the NHANES 2011-2012 cycle and lowest in 2013-2014. Past declining trends may have been misleading by not including the 2011-2012 cycle. Our prevalence estimate of $3.8 \%$ suggests that hypertensive BP among children remains an important public health issue and that the Healthy People 2020 goal of reducing this prevalence to $3.2 \%$ has thus far not been achieved (19).

Our study confirmed results of previous studies that showed overweight and obesity to be major risk factors for high BP in children $(2-5,7,9,20)$ and supports changes in the AAP guidelines to the use of BP tables based on children of healthy body weight. In our study, adjustment for weight resulted in the attenuation of prevalence differences in elevated and hypertensive BP across the sociodemographic groups examined, emphasizing the influence of weight on observed disparities in BP. Thus, future studies that examine sociodemographic differences in children's BP levels need to adjust for the child's weight in further stratified or multivariable adjusted regression analyses to more systematically examine differences across any strata under study.

Consistent with the published literature, our findings suggest that in unadjusted estimates male children, children with parent/guardian with lower levels of education, and children from families with low income levels experienced a greater burden of cardiovascular risk because of disproportionate rates of unhealthy body weight (21). Sex differences in physiologic parameters, such as total cholesterol levels, and health behaviors, such as physical activity levels, have previously been highlighted in relation to childhood obesity and could contribute to the higher unadjusted prevalence of hypertensive BP observed among males (21). Disparities in the built environment, which affect patterns of physical activity, and access to healthy foods at affordable prices are acknowledged risk factors for children of low socioeconomic status who are overweight and could contribute to the higher unadjusted prevalence of elevated BP observed in children with low levels of parent/guardian education or income $(22,23)$. Thus, through various weightrelated pathways and mediators, weight-related disparities may contribute to disparities in unadjusted prevalence of BP levels across the sociodemographic factors of sex, education, and family income.

The opinions expressed by authors contributing to this journal do not necessarily reflect the opinions of the U.S. Department of Health and Human Services, the Public Health Service, the Centers for Disease Control and Prevention, or the authors' affiliated institutions. 
The crude racial/ethnic prevalence differences detected in our study underscore the disproportionate burden of elevated BP and unhealthy weight in non-Latino Black communities $(24,25)$. Numerous factors across socioecological levels have been noted to contribute to disproportionate obesity prevalence across racial/ethnic groups $(24,25)$. Here again, we see that factors contributing to weight disparities may also contribute to BP-related disparities (23). Weight-related risk factors can be systematic and range from health care access to safety and opportunity (26). Beyond describing their existence, more action needs to be taken to disentangle and prevent the factors contributing to these disparities to achieve health equity.

In our study, racial/ethnic disparities in prevalence of elevated and hypertensive BP remained after adjusting for weight status. This indicates that factors other than body weight contribute to racial/ ethnic disparities in children's BP and that other pathways to less than optimal BP levels may begin in childhood. One such pathway is psychosocial stress, which has been extensively studied in adult populations (27). Empirical investigation of pathways (obesity-related and other) to racial/ethnic disparities in elevated BP prevalence is warranted as are interventional and policy-based efforts designed to narrow these differences and lower children's risk of subsequent cardiovascular disease. Weight disparities did not fully explain observed differences in elevated BP prevalence by sex in our study. In adult populations, sex-related BP differences are well established (28), and our findings suggest that the pathways to these sex-related BP differences may begin in childhood.

The differences we found in prevalence estimates of elevated and hypertensive BP in relation to age may be due in part to increased BP variability among young children (29) and in the use of percentile-based definitions for children aged 8 to 12 years compared with static cutoffs for children aged 13 to 17 years (30). Additionally, prevalence differences detected across age groups could be due to changes in BP associated with puberty and to the intersection of these changes with age, sex, and race/ethnicity. Further understanding is needed about how levels of BP disorders differ, and long-term follow-up data on BP levels among children are needed.

Our study highlights opportunities for reduction of elevated and hypertensive BP levels among US children. Efforts focusing on increased equity in access to care through policy changes to combat obesity in racially/ethnically and socioeconomically diverse populations should be expanded. Specific focus and efforts directed at systematic change to improve social determinants of health are also needed. Efforts to understand the causes of racial/ethnic and socioeconomic disparities and to reduce them could have shortand long-term benefits through improvements in children's health and long-term prevention into adulthood (31). Given the wellknown tracking of BP into the adult years and the strong association between elevated BP and cardiovascular and other chronic diseases, particular focus on preventing the large number of males with elevated BP from progressing to hypertension is warranted (32). Further research and risk reduction approaches should be directed to expanding BP screening in national samples of young children to improve our understanding of childhood hypertensive BP and reduce the risk of chronic diseases associated with hypertension later in life. Clinicians should be aware of socioeconomic disparities and the role of overweight highlighted in our study.

Strengths of the present study come from its use of contemporary nationally representative data and current BP screening guidelines. Although assessing subgroup differences in children's elevated and hypertensive BP may be difficult because of low case counts, we were able to combine the 4 most recent NHANES data cycles to obtain contemporary estimates across sociodemographic groups. The data analyzed in our study were collected by trained professionals who used standardized methods under controlled conditions and with quality control measures. This is important because collecting accurate BP measurements among children can be challenging (5).

Our study also has limitations. Despite the strengths inherent in the use of NHANES data, the study was limited by the data collected in that survey. Although declining response rates are of concern, NHANES has taken steps to mitigate the potential for nonresponse bias (13). Blood pressure measurements were limited to a single occasion rather than a series on 3 occasions, as is necessary for clinical diagnosis. However, previous childhood hypertension studies also used readings from a single occasion, including those providing national prevalence estimates $(1,5)$. No single measure accurately reflects socioeconomic status, and we were unable to evaluate food insecurity as a marker of socioeconomic status, or low birthweight as a potential confounder, because NHANES assesses these measures only in children aged 16 years or older. Data on other important, potentially confounding variables, including family history of hypertension, chronic kidney disease, and chronic sleep disturbance were not available.

Elevated and hypertensive BP affects US children disproportionately in various sociodemographic groups, and body weight influences these health disparities. The burden of this cardiovascular risk is higher in children who are male, non-Latino Black, or of low socioeconomic status. Age, sex, and race/ethnicity may influence BP independently of weight status. Efforts are needed to better understand and intervene on the mechanisms through which these factors interact with BP in children. Obesity and hyperten-

The opinions expressed by authors contributing to this journal do not necessarily reflect the opinions of the U.S. Department of Health and Human Services, the Public Health Service, the Centers for Disease Control and Prevention, or the authors' affiliated institutions. 
sion are preventable disorders that potentially cause lifelong harm. Continued and amplified efforts are needed related to elevated and hypertensive BP among children aimed at lowering the prevalence, decreasing disparities, and ultimately achieving health equity.

\section{Acknowledgments}

This publication is a product of a Prevention Research Center and was supported by cooperative agreement 5-U48-DP-005031 from the Centers for Disease Control and Prevention (CDC). Melissa Goulding is supported by the National Center for Advancing Translational Sciences, National Institutes of Health (NIH), through the University of Massachusetts Medical School, Center for Clinical and Translational Science predoctoral training program, grant TL1TR001454. The authors were responsible for the study design, data collection, analysis, and interpretation, and for the content of this article. The content is solely the responsibility of the authors and does not necessarily reflect the views of CDC or NIH. None of the authors have any potential, perceived, or real conflicts of interest to disclose. No copyrighted material or tools were used in this article.

\section{Author Information}

Corresponding Author: Melissa Goulding, MS, Division of Preventive and Behavioral Medicine, Population and Quantitative Health Sciences, University of Massachusetts Medical School, 368 Plantation St, Worcester, MA 01605. Telephone: 508-856-4098. Email: Melissa.Goulding@umassmed.edu.

Author Affiliations: ${ }^{1}$ Division of Preventive and Behavioral Medicine, Department of Population and Quantitative Health Sciences, University of Massachusetts Medical School, Worcester, Massachusetts. ${ }^{2}$ Division of Epidemiology, Department of Population and Quantitative Health Sciences, University of Massachusetts Medical School, Worcester, Massachusetts.

\section{References}

1. Al Kibria GM, Swasey K, Sharmeen A, Day B. Estimated change in prevalence and trends of childhood blood pressure levels in the United States after application of the 2017 AAP Guideline. Prev Chronic Dis 2019;16:E12.

2. Hales CM, Carroll MD, Fryar CD, Ogden CL. Prevalence of obesity among adults and youth: United States 2015-2016. NCHS Data Brief 2017;(288):1-8.
3. Friedemann C, Heneghan C, Mahtani K, Thompson M, Perera $\mathrm{R}$, Ward AM. Cardiovascular disease risk in healthy children and its association with body mass index: systematic review and meta-analysis. BMJ 2012;345(2):e4759.

4. Rodriguez R, Mowrer J, Romo J, Aleman A, Weffer SE, Ortiz RM. Ethnic and gender disparities in adolescent obesity and elevated systolic blood pressure in a rural US population. Clin Pediatr (Phila) 2010;49(9):876-84.

5. Flynn JT, Kaelber DC, Baker-Smith CM, Blowey D, Carroll AE, Daniels SR, et al.; Subcommittee on Screening and Management of High Blood Pressure in Children. Clinical practice guideline for screening and management of high blood pressure in children and adolescents. Pediatrics 2017; 140(3):e20171904.

6. Jackson SL, Zhang Z, Wiltz JL, Loustalot F, Ritchey MD, Goodman AB, et al. Hypertension among youths — United States, 2001-2016. MMWR Morb Mortal Wkly Rep 2018; 67(27):758-62.

7. Muntner P, He J, Cutler JA, Wildman RP, Whelton PK. Trends in blood pressure among children and adolescents. JAMA 2004;291(17):2107-13.

8. Kit BK, Kuklina E, Carroll MD, Ostchega Y, Freedman DS, Ogden CL. Prevalence of and trends in dyslipidemia and blood pressure among US children and adolescents, 1999-2012. JAMA Pediatr 2015;169(3):272-9.

9. Sorof JM, Lai D, Turner J, Poffenbarger T, Portman RJ. Overweight, ethnicity, and the prevalence of hypertension in school-aged children. Pediatrics 2004;113(3 Pt 1):475-82.

10. National High Blood Pressure Education Program Working Group on High Blood Pressure in Children and Adolescents. The fourth report on the diagnosis, evaluation, and treatment of high blood pressure in children and adolescents. Pediatrics 2004;114(2Suppl 4th Report):555-76.

11. Jackson SL, Yang EC, Zhang Z. Income disparities and cardiovascular risk factors among adolescents. Pediatrics 2018; 142(5):e20181089.

12. McGrath JJ, Matthews KA, Brady SS. Individual versus neighborhood socioeconomic status and race as predictors of adolescent ambulatory blood pressure and heart rate. Soc Sci Med 2006;63(6):1442-53.

13. Centers for Disease Control and Prevention. NHANES questionnaires, datasets, and related documentation. https:// wwwn.cdc.gov/nchs/nhanes/continuousnhanes/ default.aspx?BeginYear=2017. Accessed May 30, 2020.

14. Centers for Disease Control and Prevention. National Health and Nutrition Examination Survey. Anthropometry procedures manual. https://wwwn.cdc.gov/nchs/data/nhanes/2017-2018/ manuals/2017_Anthropometry_Procedures_Manual.pdf. Accessed July 8, 2021.

\footnotetext{
The opinions expressed by authors contributing to this journal do not necessarily reflect the opinions of the U.S. Department of Health and Human Services, the Public Health Service, the Centers for Disease Control and Prevention, or the authors' affiliated institutions.
} 
15. Centers for Disease Control and Prevention. National Health and Nutrition Examination Survey, 2015-2018: sample design and estimation procedures. Data evaluation and methods research. April 2020. https://www.cdc.gov/nchs/data/series/sr_ 02/sr02-184-508.pdf. Accessed May 30, 2020.

16. US Department of Agriculture. Supplemental Nutrition Assistance Program: SNAP eligibility. https:// www.fns.usda.gov/snap/recipient/eligibility. Accessed August 2, 2020.

17. Richardson DB, Kinlaw AC, MacLehose RF, Cole SR. Standardized binomial models for risk or prevalence ratios and differences. Int J Epidemiol 2015;44(5):1660-72.

18. Blanchette E, Flynn JT. Implications of the 2017 AAP Clinical Practice Guidelines for Management of Hypertension in Children and Adolescents: a review. Curr Hypertens Rep 2019; 21(5):35.

19. Office of Disease Prevention and Health Promotion. Healthy People 2020. Reduce the proportion of children and adolescents with hypertension. https:/www.healthypeople.gov /node/4597/data_details. Accessed August 10, 2020.

20. Gunta SS, Mak RH. Hypertension in children with obesity. World J Hypertens 2014;4(2):15-24.

21. Govindan M, Gurm R, Mohan S, Kline-Rogers E, Corriveau N, Goldberg C, et al.; University of Michigan Health System. Gender differences in physiologic markers and health behaviors associated with childhood obesity. Pediatrics 2013; 132(3):468-74.

22. Sallis JF, Conway TL, Cain KL, Carlson JA, Frank LD, Kerr J, et al. Neighborhood built environment and socioeconomic status in relation to physical activity, sedentary behavior, and weight status of adolescents. Prev Med 2018;110:47-54.

23. Walker RE, Keane CR, Burke JG. Disparities and access to healthy food in the United States: a review of food deserts literature. Health Place 2010;16(5):876-84.

24. Kirkpatrick SI, Dodd KW, Reedy J, Krebs-Smith SM. Income and race/ethnicity are associated with adherence to food-based dietary guidance among US adults and children. J Acad Nutr Diet 2012;112(5):624-635.e6.

25. Whitt-Glover MC, Taylor WC, Floyd MF, Yore MM, Yancey AK, Matthews CE. Disparities in physical activity and sedentary behaviors among US children and adolescents: prevalence, correlates, and intervention implications. J Public Health Policy 2009;30(Suppl 1):S309-34.

26. National Academies of Sciences. The state of health disparities in the United States. Washington (DC): National Academies Press; 2017. https://www.ncbi.nlm.nih.gov/books/ NBK425844/. Accessed September 30, 2020.

27. Liu M-Y, Li N, Li WA, Khan H. Association between psychosocial stress and hypertension: a systematic review and meta-analysis. Neurol Res 2017;39(6):573-80.
28. Ramirez LA, Sullivan JC. Sex differences in hypertension: where we have been and where we are going. Am J Hypertens 2018;31(12):1247-54.

29. Falkner B, Gidding SS, Portman R, Rosner B. Blood pressure variability and classification of prehypertension and hypertension in adolescence. Pediatrics 2008;122(2):238-42.

30. Bell CS, Samuel JP, Samuels JA. Prevalence of hypertension in children. Hypertension 2019;73(1):148-52.

31. Saeed A, Dixon DL, Yang E. Racial disparities in hypertension prevalence and management. A crisis control? https:// www.acc.org/latest-in-cardiology/articles/2020/04/06/08/53/ racial-disparities-in-hypertension-prevalence-and-management. Accessed July 30, 2020.

32. Chen X, Wang Y. Tracking of blood pressure from childhood to adulthood: a systematic review and meta-regression analysis. Circulation 2008;117(25):3171-80.

The opinions expressed by authors contributing to this journal do not necessarily reflect the opinions of the U.S. Department of Health and Human Services, the Public Health Service, the Centers for Disease Control and Prevention, or the authors' affiliated institutions. 


\section{Tables}

Table 1. Characteristics of Noninstitutionalized US Children Aged 8 to 17 Years, National Health and Nutrition Examination Survey (NHANES) 2011-2018

\begin{tabular}{|c|c|}
\hline Characteristic & Children (Unweighted, $N=5,971 ;$ Weighted, $N=36,612,323)^{a}$ \\
\hline \multicolumn{2}{|l|}{ Age, $y$} \\
\hline $8-9$ & 18.9 \\
\hline $10-12$ & 29.2 \\
\hline $13-15$ & 31.1 \\
\hline $16-17$ & 20.9 \\
\hline Female & 49.7 \\
\hline \multicolumn{2}{|l|}{ Race/ethnicity } \\
\hline Non-Latino White & 55.4 \\
\hline Non-Latino Black & 13.8 \\
\hline Mexican American & 14.1 \\
\hline Other Latino & 7.1 \\
\hline Non-Latino Asian & 4.1 \\
\hline Other ${ }^{b}$ & 5.5 \\
\hline \multicolumn{2}{|l|}{ Highest level parent/guardian education } \\
\hline$\geq$ College graduate & 28.8 \\
\hline High school diploma/GED/some college & 52.6 \\
\hline$<$ High school diploma & 18.5 \\
\hline \multicolumn{2}{|l|}{ Family income $^{c}$} \\
\hline High & 30.1 \\
\hline Medium & 39.2 \\
\hline Low & 30.7 \\
\hline \multicolumn{2}{|l|}{ Weight status } \\
\hline BMI percentile, median (IQR) & $73.3(42.7-93.0)$ \\
\hline Healthy weight (BMI percentile <85) & 62.5 \\
\hline Overweight (BMI percentile $\geq 85$ to $<95$ ) & 17.0 \\
\hline Obesity (BMI percentile $\geq 95$ ) & 20.6 \\
\hline
\end{tabular}

Abbreviation: BMI, body mass index; IQR, interquartile range.

${ }^{a}$ Values are weighted percentage unless otherwise indicated.

${ }^{\mathrm{b}}$ Includes American Indian or Alaska Native, Native Hawaiian or Pacific Islander, and mixed race.

${ }^{c}$ Determined by family poverty income ratio (PIR): family income divided by Department of Health and Human Services poverty guidelines (specific to family size, year, and state of residence). High $=$ PIR $>3.5$, medium $=$ PIR $\geq 1.3,<3.5$; low $=$ PIR $<1.3$. 
Table 2. Prevalence of Elevated and Hypertensive Blood Pressure ${ }^{a}$ Among US Children Aged 8 to 17 Years $(N=36,612,323)^{b}$, by Biennial Cycle, National Health and Nutrition Examination Survey (NHANES) 2011-2018

\begin{tabular}{|l|r|r|}
\hline NHANES cycle & Elevated Blood Pressure Prevalence, \% (95\% Cl) & \multicolumn{1}{|c|}{ Hypertensive Blood Pressure Prevalence \%, (95\% Cl) } \\
\hline $2011-2012$ & $8.3(6.4-10.7)$ & $4.6(3.5-6.1)$ \\
\hline $2013-2014$ & $6.0(4.6-8.0)$ & $2.6(1.7-3.8)$ \\
\hline $2015-2016$ & $8.2(6.6-10.3)$ & $4.3(2.9-6.3)$ \\
\hline $2017-2018$ & $6.2(4.2-9.3)$ & $3.9(2.9-5.3)$ \\
\hline
\end{tabular}

${ }^{a}$ Hypertensive and elevated blood pressure determined by 2017 American Academy of Pediatrics guidelines. Hypertensive: blood pressure percentile $\geq 95$ or average blood pressure $\geq 130 / 80 \mathrm{~mm} \mathrm{Hg}$ (whichever was lower) for children aged 8-12 years and $\geq 130 / 80 \mathrm{~mm}$ Hg for children aged $\geq 13$ years. Elevated blood pressure: $\geq 90$ th percentile to $<95$ th percentile or $120 /<80 \mathrm{~mm} \mathrm{Hg}$ to $<95$ th percentile (whichever is lower) for children aged $8-12$ years and $120 /<80$ to $129 /<80$ $\mathrm{mm} \mathrm{Hg}$ for children aged 13 to 17 years.

${ }^{\mathrm{b}}$ Unweighted, $\mathrm{N}=5,971$. 
Table 3. Prevalence of Elevated and Hypertensive Blood Pressure ${ }^{a}$ by Sociodemographic Characteristics, US Children Aged 8 to 17 Years $(\mathrm{N}=36,612,323)^{b}$, National Health and Nutrition Examination Survey (NHANES) 2011-2018

\begin{tabular}{|c|c|c|c|c|c|c|}
\hline \multirow[b]{2}{*}{ Characteristic } & \multicolumn{3}{|c|}{ Elevated blood pressure } & \multicolumn{3}{|c|}{ Hypertensive blood pressure } \\
\hline & $\begin{array}{l}\text { Prevalence, \% } \\
\quad(95 \% \mathrm{Cl})\end{array}$ & $\begin{array}{c}\text { Crude Prevalence } \\
\text { Difference }(95 \% \mathrm{Cl})\end{array}$ & $\begin{array}{c}\text { Prevalence } \\
\text { Difference Adjusted } \\
\text { for Overweight/ } \\
\text { Obesity, \% (95\% Cl) }\end{array}$ & $\begin{array}{l}\text { Prevalence, \% } \\
\quad(95 \% \mathrm{Cl})\end{array}$ & $\begin{array}{c}\text { Crude Prevalence } \\
\text { Difference }(95 \% \mathrm{Cl})\end{array}$ & $\begin{array}{c}\text { Prevalence } \\
\text { Difference Adjusted } \\
\text { for Overweight/ } \\
\text { Obesity, \% (95\% Cl) }\end{array}$ \\
\hline \multicolumn{7}{|l|}{ BMI percentile ${ }^{c}$} \\
\hline $\begin{array}{l}\text { Healthy weight, } \\
<85\end{array}$ & 4.9 (4.1 to 5.9$)$ & Reference & \multirow[t]{3}{*}{$\mathrm{NA}$} & $2.2(1.7$ to 2.8$)$ & Reference & \multirow[t]{3}{*}{ NA } \\
\hline Overweight, $\geq 85$ to $<95$ & 9.2 (7.1 to 12.0$)$ & 4.3 (1.8 to 6.8$)$ & & 4.1 (2.7 to 6.1$)$ & 1.9 (0.3 to 3.5$)$ & \\
\hline Obesity, $\geq 95$ & $12.7(10.7$ to 15.1$)$ & 7.8 (5.7 to 9.9$)$ & & 8.6 (6.9 to 10.9$)$ & $6.4(4.3$ to 8.6$)$ & \\
\hline \multicolumn{7}{|l|}{ Age, $y$} \\
\hline $8-9$ & 5.9 (4.4 to 8.0$)$ & Reference & Reference & 6.0 (4.6 to 8.0$)$ & Reference & Reference \\
\hline $10-12$ & 4.3 (3.2 to 6.0$)$ & $-1.6(-3.6$ to 0.5$)$ & $-1.7(-3.6$ to 0.3$)$ & 4.0 (3.0 to 5.3$)$ & $-2.1(-4.1$ to 0.02$)$ & $-1.5(-3.6$ to 0.7$)$ \\
\hline $13-15$ & 7.0 (5.6 to 8.8$)$ & $1.1(-1.2$ to 3.4$)$ & $0.7(-1.5$ to 2.8$)$ & $2.0(1.4$ to 2.8$)$ & $-4.1(-5.9$ to -2.3$)$ & $-3.8(-5.6$ to -2.0$)$ \\
\hline $16-17$ & $12.8(10.4$ to 15.8$)$ & 6.9 (3.7 to 10.2$)$ & $6.3(3.2$ to 9.4$)$ & 4.5 (3.2 to 6.3$)$ & $-1.6(-3.9$ to 0.8$)$ & $-1.4(-3.6$ to 0.7$)$ \\
\hline \multicolumn{7}{|l|}{ Sex } \\
\hline Female & 4.9 (3.9 to 6.1$)$ & Reference & Reference & $3.0(2.2$ to 4.1$)$ & Reference & Reference \\
\hline Male & 9.6 (8.1 to 11.2$)$ & 4.6 (2.8 to 6.5$)$ & $4.6(2.7$ to 6.4$)$ & 4.7 (3.7 to 5.9$)$ & $1.7(0.2$ to 3.2$)$ & $1.3(-0.2$ to 2.8$)$ \\
\hline \multicolumn{7}{|l|}{ Race/ethnicity } \\
\hline Non-Latino White & 6.3 (5.1 to 7.9$)$ & Reference & Reference & $3.2(2.4$ to 4.3$)$ & Reference & Reference \\
\hline Non-Latino Black & 10.4 (8.8 to 12.1$)$ & $4.0(2.1$ to 5.9$)$ & $4.0(2.2$ to 5.8$)$ & 4.4 (3.3 to 5.8 ) & $1.2(-0.3$ to 2.7$)$ & $0.5(-0.8$ to 1.9$)$ \\
\hline Mexican American & 8.4 (6.8 to 10.5$)$ & 2.1 (-0.1 to 4.3$)$ & $1.6(-0.5$ to 3.7$)$ & 5.2 (3.9 to 6.8 ) & 2.0 (0.1 to 3.9$)$ & $1.3(-0.4$ to 2.9$)$ \\
\hline Other Latino $^{\mathrm{d}}$ & 8.0 (6.0 to 10.6$)$ & $1.7(-0.9$ to 4.3$)$ & $1.7(-0.7$ to 4.1$)$ & 3.9 (2.4 to 6.2$)$ & 0.7 (-1.2 to 2.6$)$ & $0.3(-1.5$ to 2.0$)$ \\
\hline Non-Latino Asian ${ }^{\mathrm{d}}$ & 4.6 (2.9 to 7.4 ) & $-1.7(-4.5$ to 1.0$)$ & $-0.2(-2.8$ to 2.5$)$ & 4.3 (2.8 to 6.4 ) & $1.1(-0.7$ to 2.9$)$ & $1.5(-0.2$ to 3.1$)$ \\
\hline Other ${ }^{\mathrm{d}, \mathrm{e}}$ & 6.7 (4.2 to 10.7$)$ & $0.4(-2.9$ to 3.7$)$ & $0.2(-2.7$ to 3.1$)$ & 5.5 (3.5 to 8.6) & $2.3(-0.4$ to 5.1$)$ & $1.9(-0.6$ to 4.4$)$ \\
\hline \multicolumn{7}{|l|}{ Family education } \\
\hline$\geq$ College graduate & 5.4 (4.0 to 7.4$)$ & Reference & Reference & 3.9 (2.7 to 5.5$)$ & Reference & Reference \\
\hline $\begin{array}{l}\text { High school diploma/ } \\
\text { GED/some college }\end{array}$ & 8.0 (6.8 to 9.4$)$ & 2.5 (0.6 to 4.5$)$ & $1.6(-0.3$ to 3.5$)$ & 3.8 (3.0 to 4.9 ) & $-0.02(-1.7$ to 1.7$)$ & $-0.7(-2.4$ to 0.9$)$ \\
\hline <High school diploma & $8.0(6.5$ to 9.9$)$ & 2.6 (0.4 to 4.8$)$ & 2.1 (0.0 to 4.3 ) & 3.8 (2.8 to 5.2$)$ & $-0.06(-1.8$ to 1.7$)$ & $-0.5(-2.3$ to 1.3$)$ \\
\hline \multicolumn{7}{|l|}{ Family income ${ }^{f}$} \\
\hline High & 5.8 (4.2 to 7.9$)$ & Reference & Reference & 3.1 (2.1 to 4.5$)$ & Reference & Reference \\
\hline
\end{tabular}

Abbreviation: NA, not applicable.

${ }^{a}$ Hypertensive and elevated blood pressure determined by 2017 American Academy of Pediatrics guidelines. Hypertensive: blood pressure percentile $\geq 95$ or average blood pressure $\geq 130 / 80 \mathrm{~mm} \mathrm{Hg}$ (whichever was lower) for children aged $8-12$ years and $\geq 130 / 80 \mathrm{~mm} \mathrm{Hg}$ for children aged $\geq 13$ years. Elevated blood pressure: $\geq 90$ th percentile to $<95$ th percentile or $120 /<80 \mathrm{~mm} \mathrm{Hg}$ to $<95$ th percentile (whichever is lower) for children aged $8-12$ years and $120 /<80$ to $129 /<80$ $\mathrm{mm} \mathrm{Hg}$ for children aged 13 to 17 years.

${ }^{\mathrm{b}}$ Unweighted, $\mathrm{N}=5,971$.

${ }^{\mathrm{c}} \mathrm{BMI}$ (weight in $\mathrm{kg} / \mathrm{height}$ in $\mathrm{m}^{2}$ ) as percentile according to the Centers for Disease Control and Prevention 2000 growth charts.

${ }^{\mathrm{d}}$ Had fewer than 30 participants; therefore, did not meet NHANES reporting standards in the hypertensive category.

e Includes American Indian or Alaska Native, Native Hawaiian or Pacific Islander, and mixed race.

${ }^{f}$ Determined by family poverty income ratio; family income divided by Department of Health and Human Services poverty guidelines (specific to family size, year and sate of residence). High PIR $=>3.5$, medium $\mathrm{PIR}=\geq 1.3$ to $<3.5$, low $\mathrm{PIR}=<1.3$. 
(continued)

Table 3. Prevalence of Elevated and Hypertensive Blood Pressure ${ }^{a}$ by Sociodemographic Characteristics, US Children Aged 8 to 17 Years $(\mathrm{N}=36,612,323)^{b}$, National Health and Nutrition Examination Survey (NHANES) 2011-2018

\begin{tabular}{|c|c|c|c|c|c|c|}
\hline \multirow[b]{2}{*}{ Characteristic } & \multicolumn{3}{|c|}{ Elevated blood pressure } & \multicolumn{3}{|c|}{ Hypertensive blood pressure } \\
\hline & $\begin{array}{l}\text { Prevalence, \% } \\
\quad(95 \% \mathrm{Cl})\end{array}$ & $\begin{array}{l}\text { Crude Prevalence } \\
\text { Difference }(95 \% \mathrm{Cl})\end{array}$ & $\begin{array}{c}\text { Prevalence } \\
\text { Difference Adjusted } \\
\text { for Overweight/ } \\
\text { Obesity, } \%(95 \% \mathrm{Cl})\end{array}$ & $\begin{array}{l}\text { Prevalence, \% } \\
\quad(95 \% \mathrm{Cl})\end{array}$ & $\begin{array}{l}\text { Crude Prevalence } \\
\text { Difference }(95 \% \mathrm{Cl})\end{array}$ & $\begin{array}{c}\text { Prevalence } \\
\text { Difference Adjusted } \\
\text { for Overweight/ } \\
\text { Obesity, \% (95\% Cl) }\end{array}$ \\
\hline Medium & $7.8(6.5$ to 9.2$)$ & $2.0(-0.3$ to 4.2$)$ & $1.3(-1.1$ to 3.6$)$ & $4.0(3.1$ to 5.2$)$ & $0.9(-0.7$ to 2.6$)$ & $0.4(-0.9$ to 1.7$)$ \\
\hline Low & $8.4(7.3$ to 9.6$)$ & $2.2(0.4$ to 4.0$)$ & $1.4(-0.4$ to 3.2$)$ & 4.6 (3.6 to 5.8$)$ & $1.4(-0.2$ to 3.0$)$ & $0.6(-0.8$ to 2.1$)$ \\
\hline
\end{tabular}

Abbreviation: NA, not applicable.

${ }^{a}$ Hypertensive and elevated blood pressure determined by 2017 American Academy of Pediatrics guidelines. Hypertensive: blood pressure percentile $\geq 95$ or average blood pressure $\geq 130 / 80 \mathrm{~mm} \mathrm{Hg}$ (whichever was lower) for children aged $8-12$ years and $\geq 130 / 80 \mathrm{~mm} \mathrm{Hg}$ for children aged $\geq 13$ years. Elevated blood pressure: $\geq 90$ th percentile to $<95$ th percentile or $120 /<80 \mathrm{~mm} \mathrm{Hg}$ to $<95$ th percentile (whichever is lower) for children aged $8-12$ years and $120 /<80$ to $129 /<80$ $\mathrm{mm} \mathrm{Hg}$ for children aged 13 to 17 years.

${ }^{\mathrm{b}}$ Unweighted, $\mathrm{N}=5,971$.

${ }^{\mathrm{C}} \mathrm{BMI}$ (weight in $\mathrm{kg} / \mathrm{height}$ in $\mathrm{m}^{2}$ ) as percentile according to the Centers for Disease Control and Prevention 2000 growth charts.

${ }^{d}$ Had fewer than 30 participants; therefore, did not meet NHANES reporting standards in the hypertensive category.

e Includes American Indian or Alaska Native, Native Hawaiian or Pacific Islander, and mixed race.

${ }^{f}$ Determined by family poverty income ratio; family income divided by Department of Health and Human Services poverty guidelines (specific to family size, year and sate of residence). High PIR $=>3.5$, medium $\mathrm{PIR}=\geq 1.3$ to $<3.5$, low $\mathrm{PIR}=<1.3$.

The opinions expressed by authors contributing to this journal do not necessarily reflect the opinions of the U.S. Department of Health and Human Services, the Public Health Service, the Centers for Disease Control and Prevention, or the authors' affiliated institutions. 